\title{
Erfolge und Probleme der modernen (Mainstream-)Makroökonomik: Replik zum Kommentar von Peter Spahn
}

\author{
Rüdiger Bachmann
}

Online publiziert: 22. Februar 2019

(C) List-Gesellschaft e.V. 2019

Zunächst einmal bedanke ich mich bei Peter Spahn für seinen Kommentar. In dieser Replik werde ich drei Dinge tun: 1) einige nach meinem Dafürhalten faktische Fehler in Peter Spahns Kommentar korrigieren; 2) Peter Spahns Sichtweise der Funktion von Theorie und Empirie und deren Wechselwirkung sowie zum Pluralismus in der Ökonomik herausarbeiten und einen wenn auch nicht ganz fundamentalen, so doch substantiellen Dissens konstatieren; 3) auf Peter Spahns Kritik an der Neukeynesianischen Makroökonomik eingehen und ihr partiell zustimmen, dann aber fragen, warum Peter Spahn so gut wie nicht auf den zweiten (und dritten) Teil des Hauptaufsatzes eingegangen ist, die einige Kritik an der Neukeynesianischen Makroökonomik enthalten.

1 .

- Die Behauptung, das Niveau der gesamtwirtschaftlichen Beschäftigung sei in Neukeynesianischen Modellen angebotsbedingt, ist bestenfalls unterkomplex. Im einfachsten Fall mit Monopol- und Preissetzungsmacht auf den Gütermärkten, aber ohne Monopol- und Lohnsetzungsmacht der „Gewerkschaften“, ist das gesamtwirtschaftliche Produktionsniveau rein nachfragebedingt. Das ergibt sich zwingend aus der Logik der monopolistischen Konkurrenz in den Modellen, bei der die Firmen wegen rigider Preise so lange ihr Angebot ausweiten könnten, bis sie keine Gewinne mehr machen. Dieses nachfragedeterminierte Produktionsniveau bestimmt dann durch die Produktionsfunktion auch das

This reply refers to the commentary article available online at https://doi.org/10.1007/s41025-01900144-5.

R. Bachmann $(\triangle)$

3026 Jenkins Nanovic Hall, Department of Economics, University of Notre Dame, Notre

Dame, IN 46556, USA

E-Mail: rbachman@nd.edu 
gesamtwirtschaftliche Beschäftigungsniveau. Ähnliches gilt für den Fall von Monopol- und Lohnsetzungsmacht der „Gewerkschaften“.

- Warum Peter Spahn behauptet, die Arbeitslosigkeit in Neukeynesianischen Modellen sei Sucharbeitslosigkeit, ist nicht nachvollziehbar. Im einfachsten Neukeynesianischen Modell gibt es zunächst einmal gar keine Arbeitslosigkeit (wofür man das Modell gerne kritisieren kann). Es gibt zwar Erweiterungen des Modells, in denen Sucharbeitslosigkeit eingefügt wird (etwa Christiano et al. 2016; Gali et al. 2011, nennt noch andere Beiträge in diese Richtung), aber es geht auch anders, etwa über Marktmacht (Gali et al. 2011). Peter Spahns Schlussfolgerungen über Neukeynesianische Modelle sind bei diesen faktischen Fehlern daher mit Vorsicht zu behandeln.

- Peter Spahn stellt richtig fest, dass Neukeynesianische Modelle oft in der Totalen durch sogenannte full information Schätzungen mit den Daten konfrontiert werden. Das ist aber nicht die einzige Konfrontation dieser Modelle und ihrer Bestandteile mit den Daten. Jedes einzelne Modellelement, jeder einzelne Teilmechanismus wird auch separat an die Daten herangetragen, ein Großteil der neueren empirischen Methoden und Erkenntnisse dazu wurde im Hauptaufsatz genannt. Es ist Peter Spahn, der hier Etikettenschwindel betreibt, zumal er ja in seinem Kommentar die Beschäftigung mit dieser Mikroevidenz auch wiederum als nicht immer hilfreich zu erachten scheint, was im nächsten Punkt noch genauer zu beleuchten sein wird.

- Es ist falsch, dass die gleichzeitige Analyse von wichtigen Friktionen in der modernen Makroökonomik keine Rolle spielt. Der Hauptaufsatz enthält zu diesem Thema einen eigenen Abschnitt.

2. Peter Spahn ist letztlich ein Theoretiker mit einer Vorliebe für Aggregatsmodelle, die etwa den von Blanchard (2018) so genannten toy models entsprechen. Als Empirie scheint Peter Spahn hauptsächlich seine eigene Intuition zuzulassen (,Die NKM ist [...] intuitiv wenig überzeugend"), jedenfalls geht er auf die im Hauptaufsatz diskutierte statistische oder einfach datenbasierte Evidenz kaum ein, oder empfindet sie als trivial (die stärker werdende Asymmetrie von idiosynkratischem Einkommensrisiko in Rezessionen und die Zustandsabhängigkeit von Fiskalmultiplikatoren werden ausdrücklich genannt). Mit traditioneller Theorie habe er das schon immer gewusst. Gut für Peter Spahn. Aber letztlich sollte doch für einen Wissenschaftler, der etwas über die Welt aussagen will, der Beweis in den Daten liegen, nicht in der Theorie, gleichgültig, ob traditionell oder modern. Warum Peter Spahn Sucharbeitslosigkeit ablehnt, bleibt unklar. Die Fülle von Daten, die in den letzten Jahrzehnten über den Arbeitsmarkt gesammelt wurden, und im Hinblick auf die Such- und Matchingmodelle entworfen wurden, scheint ihn nicht zu interessieren. Über Erwartungen weiß Peter Spahn, dass nur adaptive Erwartungen begründbar sind. Dabei ignoriert er den (wenn auch kurzen) Abschnitt zur empirischen Erwartungsbildungs- und Erwartungsauswirkungsforschung im Hauptaufsatz völlig. Ricardianische Äquivalenz sei selten beobachtet worden - woher weiß Peter Spahn das? Im Übrigen verkennt er den Status des Ricardianischen Äquivalenztheorems in der Ökonomik. Es ist natürlich wie jedes mathematische Theorem, das man in der Wirklichkeit zu finden hofft, in einem bestimmten, trivialen Sinne falsch. Ricardianische Äquivalenz ist vielmehr ein Mechanismus, der 
beschreibt, was bei reinen Staatsfinanzierungsänderungen geschieht, wenn Wirtschaftssubjekte in die Zukunft schauen. Tun sie das überhaupt nicht? Man kann das empirisch untersuchen, und die Antwort lautet meistens - partiell. Was Peter Spahn gegen eine Befruchtung der Mikroökonomik durch makroökonomische Evidenz hat (er nennt das etwas spöttisch „Makrofundierung der Mikroökonomie“), bleibt ebenfalls unklar: was spricht eigentlich gegen eine wechselseitige Befruchtung der Teildisziplinen einer einheitlichen Ökonomik?

Dagegen schätzt Peter Spahn einfache Modelle, einfache Theorie, sogar das einfache Robinson-Crusoe-Modell sehr, ähnlich wie Caballero (2010). Ebenfalls in einer antiempirischen Wendung, steht er auf Mikrodaten beruhenden Modellkomplikationen (wie Heterogenität) skeptisch gegenüber, unter anderem, weil sie die Modelle noch unhandlicher machten und die wissenschaftliche Kommunikation erschwerten. Das aber scheint mir doch sehr im Auge des Betrachters im Lichte des jeweils erworbenen wissenschaftlichen Humankapitals zu liegen. Ich zum Beispiel finde manche einfachen ad hoc Aggregatsmodelle, die Peter Spahn wahrscheinlich im Schlaf beherrscht, unverständlich. Und was wäre überhaupt das Kriterium dafür, wann man und wann man nicht aus den Mikrodaten lernen darf? Schließlich scheint Peter Spahn gar nicht so viel von Pluralität in der Ökonomik zu halten. Was die von mir im Hauptaufsatz konstatierte und begrüßte zunehmende und noch zu verbessernde soziologische Pluralität angeht, so scheint er diese für nicht wirklich wichtig zu halten, ebenso wenig wie Interdisziplinarität (bei letzterem kann ich partiell zustimmen; ebenso wie seiner Kritik an Teilen der Ökonomikkritik durch die Plurale Bewegung). Eine behavioristische Wende der Ökonomik lehnt er ab, mit Ausnahme der rationalen Erwartungen. Das bleibt aber aus zweierlei Gründen etwas dunkel, denn einer Kritik am homo oeconomicus kann er nicht viel abgewinnen, was zwar nicht per se inkompatibel mit einer Kritik an rationalen Erwartungen ist, die Peter Spahn dann aber doch behavioristisch formuliert (,Die Wirtschaftsbürger sind wohl eher nicht in der Lage, Makromodelle per Forward Solving zur Erwartungsbildung zu nutzen."); hier hätte man doch gerne mehr erfahren. Zweitens bleibt, wie schon angedeutet, seine einfache Deklaration, dass man eben adaptive Erwartungen nehmen müsse, empirisch untermotiviert. Insgesamt bin ich in meinem Hauptaufsatz doch eigentlich pluraler: ich räume ja ausdrücklich wie Blanchard (2018) den einfachen toy models (man kann den Namen gerne ändern, um ihre Bedeutung adäquater widerzuspiegeln) eine wichtige Rolle ein, ebenso wie vielen anderen Modelltypen. Peter Spahn ist da wesentlich monistischer. Hier wäre eine etwas detailliertere Auseinandersetzung mit dem Schlussteil des Hauptaufsatzes doch wünschenswert gewesen, statt einer nur kurzen abtuenden Erwähnung desselben im Einleitungsparagraphen des Kommentars.

3. Ich bin zwar ein Fan der modernen Makroökonomik, aber nicht unbedingt ein Fan der Neukeynesianischen Makroökonomik. Nichtsdestotrotz musste ich jene natürlich als Folie, vor der die weiteren Entwicklungen ablaufen, darstellen. Und genauso natürlich verteidige ich die Neukeynesianische Makroökonomik gegenüber ungerechtfertigter Kritik. Es scheint, hier ist Peter Spahn einem Missverständnis erlegen, dass sich der Verfasser des Hauptaufsatzes allerdings selbst zuzuschreiben hat und das er nun hofft, mit dieser Replik korrigieren zu können: 
nahezu der gesamte zweite Teil des Hauptaufsatzes kann und soll als Kritik an, mindestens aber als Test der Neukeynesianischen Makroökonomik gelesen werden. Umso mehr bedauere ich es, dass Peter Spahn diesen nicht rezipiert hat. Er hätte nämlich wahrscheinlich einiges gefunden, das ihm gefallen hätte: eine Infragestellung der Konsum-Eulergleichung etwa im Abschnitt zur empirischen Erwartungsforschung, in den theoretischen Ausführungen zu HANK und im Abschnitt über Nachfrageschocks. Einkommen und Einkommenserwartungen spielen in diesen Modellen eine viel größere Rolle. Einschub: ich halte die KonsumEulergleichung auch für das problematischste Element der Neukeynesianischen Makroökonomik. Auch die von Peter Spahn betonten Einkommensbildungswirkungen der Investitionen ließen sich in den neuen Nachfrageschockmodellen darstellen. Dies gilt aber auch umgekehrt für die andere Altkeynesianische Hypothese der Einkommenswirkungen auf die Investitionen. Natürlich wird Peter Spahn sagen, dass hatten wir auch in einem alten (modifizierten) ISLM Modell schon, aber ich will es genauer wissen: was ist genau der Mechanismus, der dazu führt, dass Investitionen Einkommenswirkungen haben und umgekehrt? Handelt es sich hier um finanzielle Friktionen, die cash flow Variablen wichtig werden lassen, oder um animal spirits, die auf Einkommenserwartungen beruhen, etc.? Ich kann jedenfalls nicht verstehen, warum man das nicht genauer wissen wollte.

\section{Literatur}

Blanchard, O. (2018). On the future of macroeconomic models. Oxford Review of Economic Policy, $34(1-2), 43-54$.

Caballero, R. (2010). Macroeconomics after the crisis: Time to deal with the pretense-of-knowledge syndrome. Journal of Economic Perspectives, 24(4), 85-102.

Christiano, L., Eichenbaum, M., \& Trabandt, M. (2016). Unemployment and business cycles. Econometrica, 84(4), 1523-1569.

Gali, J., Smets, F., \& Wouters, R. (2011). Unemployment in an estimated New Keynesian model. NBER Macroeconomics Annual, 26, 329-360. 